\title{
Ausbildung und Erwartungshaltung
}

\section{Sehr geehrte Leserin, sehr geehrter Leser,}

werden Geologiestudenten zeitgemäß ausgebildet? Wird in der Angewandten Geologie das gelehrt, was in Wirtschaftsunternehmen an Wissen von einem Geologen erwartet wird? Erfolgt die spezielle Ausbildung zum Hydrogeologen fundiert und umfassend?

Eine Antwort auf diese Fragen kann sicherlich nicht allumfassend gegeben werden, da sie von der jeweiligen Sichtweise abhängig ist.

Ein Geologieprofessor wird dieAusbildung seiner Studenten im Großen und Ganzen als zeitgemäß einstufen, eventuell hier und da Verbesserungen für möglich halten.

Auffällig ist die hohe Anzahl der in den letzten Jahren bereits geänderten oder zur Änderung anstehenden Studienordnungen. Es zeigt, daß die Straffung des Studiums sowie eine zumindest teilweise neue Wichtung der Schwerpunkte notwendig war bzw. ist, wobei im allgemeinen eine höhere Stundenzahl in den Grundlagenfächern Mathematik,Physik und Chemie angesetzt wird.

Betrachtet man die Gesamtstundenzahlen der alten und der reformierten Studienordnungen, kann jedoch von einer Kürzung der Pflichtstunden in den meisten Fällen nicht die Rede sein.

Eine grundlegende Reform des Geologiestudiums ist zur Zeit nicht zu erkennen, eher eineVerschulung des Studienganges. Die Entwicklung scheint zur Zeit weg von der freien Kurswahl und hin zu Modulangeboten mit vorgegebenen Kursen und Reihenfolgen zu gehen.

Für die Hydrogeologie-Professoren ist es sicherlich nicht einfach zu entscheiden und in ihrem Kollegenkreis durchzusetzen, was aus dem generellen geowissenschaftlichen Kursangebot für einen Hy- drogeologen notwendig oder aber entbehrlich ist.

Genügen die Grundlagen der Paläontologie und der Mineralogie? Kann man ganz darauf verzichten?

Was erwarten Behörden und Wirtschaftsunternehmen an allgemeinen und Spezialkenntnissen?

Geologische Landesämter z. B. wollen, pauschal betrachtet, Geologen alten Stils. Ist ausdrücklich ein Angewandter Geologe oder speziell ein Hydrogeologe erwünscht, soll dieser die Spezialkenntnisse zusätzlich mitbringen. Außerdem wird eine kurze Studiendauer vorausgesetzt. Und was für eine Ausbildung erwarten Wirtschaftsunternehmen von Hydrogeologen? Die Antwort auf diese Frage hängt einerseits von der Größe des Unternehmens und andererseits vom Markt mit seinen aktuell zu bearbeitenden Problemen ab. Allen gemein ist der Wunsch nach unternehmerischer Sichtweise und die Bereitschaft, Verantwortung zu übernehmen. Äußerungen von Managern, die Angewandte Geologen nur als Lieferanten von Zahlen (oder Größen) einschätzen, zeigen meines Erachtens, daß beide Seiten nicht die Fachproblematik des anderen erkennen und in ihre Sichtweise umsetzen können. Man kann mit Bedauern über solche Äußerungen hinweggehen und sie als Einzelfälle abtun. Sie zeigen jedoch exemplarisch ein Defizit im Verständnis zwischen Angewandten Geologen und Managern. Deren Ausbildung beruht zumeist auf einer juristischen oder betriebswirtschaftlichen bzw.volkswirtschaftlichen Grundlage. Um juristisch ausgebildeten Entscheidungsträgern eine Maßnahme oder deren mögliche Alternativen nahezubringen, sollte deren Denkund Entscheidungsweise nachvollzogen werden können.

Wenn auf der anderen Seite von einem Angewandten Geologen eine Sanie- rungsmaßnahme vorgeschlagen wird, die einer Kosten - Nutzenanalyse nicht standhält, zeigt dies fehlende Einblicke in betriebswirtschaftliche Grundkenntnisse.

Diese Art von Ausbildung fehlt im Angebot an den Deutschen Universitäten. Eine echte Studienreform müßte als Wahl- oder Wahlpflichtfächer Jura, Betriebswirtschaft und Informatik für alle Angewandten Geowissenschaftler berücksichtigen.

Konsequenterweise muß dann auf andere Studieninhalte verzichtet werden, und es ergeben sich daraus eine Reihe von unbequemen Fragen:

Was soll als Grundausbildung jeder Geologe wissen, was jeder Hydrogeologe? Kann z. B. ein Hydrogeologe ohne Grundkenntnisse der Ingenieurgeologie und der Lagerstättenkunde leben? Ist es nötig, Sedimentologie,Bodenkunde, Geophysik oder Paläontologie belegt zu haben? Sind Spezialisten in Spezialdisziplinen langfristig auf dem Markt marktfähig? Kann und soll dieAusbildung auf kurzfristige Marktänderungen Rücksicht nehmen?

Mir erscheint eine verstärkte Ausbildung zur Problemerkennung und -beschreibung in Arbeitsteams notwendig zu sein, verbunden mit einem juristischen oder wirtschaftlichen Wahlfach. Dies sehe ich als die beste Möglichkeit, um langfristig zu verhindern, das (Hydro)Geologen als naturwissenschaftliche Hilfsarbeiter abqualifiziert werden.

Damit sie weiterhin für unsere Umwelt die optimalen (und leider meist nicht die besten) Problemlösungen vorschlagen und auch durchsetzen können.

\section{Dr. Holger Knoke}

Editor Nachrichten 